\title{
Mit den Menschen für die Menschen planen. Praktikentheorien, Planungskoalitionen und die Treffpunkte der Drogen- und Alkoholabhängigen in Kiel-Gaarden
}

\author{
Lars Kraehnke \\ Eingegangen: 5. Oktober 2020 - Angenommen: 28. Mai 2021 - Online veröffentlicht: 10. Juni 2021
}

\section{Zusammenfassung}

Am Beispiel des kollektiven Alkoholkonsums einer Szene von Drogen- und Alkoholabhängigen im Kieler Stadtteil Gaarden wurde untersucht, inwiefern auf den ersten Blick banal erscheinende Praktiken wie das gemeinsame Konsumieren alkoholhaltiger Getränke einen Beitrag zur sozialen und materiellen Ausgestaltung von Szenetreffpunkten im öffentlichen Raum leisten. Klassischerweise wird die Gestaltung dieser Räume als zentrale Aufgabe von Planung auf verschiedenen Maßstabsebenen angesehen. Räumlich wird der Planungsprozess dabei in der Regel in den Büros der Verwaltung, den Plenarsälen der Politik oder an den sprichwörtlichen Runden Tischen diverser Beteiligungsverfahren verortet. Im vorliegenden Beitrag wird hingegen ein anderes Planungsverständnis vorgeschlagen. Unter Rückgriff auf aktuelle Theorieentwicklungen im Bereich raumbezogener Praktikentheorien werden sowohl die Praktiken der formal autorisierten Planerinnen und Planer als auch diejenigen der Szenemitglieder als konstitutiver Bestandteil des Planungsprozesses aufgefasst. Der Planungsprozess verlagert sich damit zu einem nicht unwesentlichen Teil an die Szenetreffpunkte vor Ort. Die Szenemitglieder leisten durch ihr Handeln einen Beitrag zum Planungsprozess und werden in gewisser Weise selbst zu Planerinnen und Planern, die mittels kollektiver Konsumpraktiken ihren Treffpunkt mitgestalten.

\footnotetext{
$\bowtie$ Lars Kraehnke, Christoph-Bernhard-Graben 66a, 48163 Münster, Deutschland lars@kraehnke.de
}

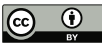
( 2021 Kraehnke; licensee oekom verlag. This Open Access article is published under a Creative Commons Attribution 4.0 International License.
Schlüsselwörter: Praktiken - Planung Planungskoalitionen - Treffpunkte - Alkoholkonsum - Kiel

Planning with the people for the people. Theories of practice, planning coalitions and the gathering places of drug and alcohol addicts in Kiel-Gaarden

\begin{abstract}
This paper shows how publicly accessible places like the gathering places of street drinkers and drug addicted are being shaped and defined by practices as banal and mundane as the collective consumption of alcoholic drinks. In a classical view it is the role of spatial planners on different scales to design these places and to specify how they can be used. In scientific as well as societal debates planning processes are therefore usually located in administration offices, plenary halls of the government or citizen-participation processes. On the contrary, this paper proposes a different understanding of spatial planning. Following current practice-theoretical debates the practices of planners as well as the practices the members of the drinking community perform are conceptualised as a constitutive part of the planning process. As a consequence the planning process itself does not only take place in offices and plenary halls. Instead the site of planning shifts (at least partly) to the gathering places in public space and the street drinkers themselves become planners, who shape their places through the performance of collective drinking-practices.
\end{abstract}

Keywords: Practices - Planning - Planning coalitions Gathering places - Alcohol consumption - Kiel 


\section{Einleitung}

Kollektiver Alkoholkonsum in öffentlichen Räumen wird in gesellschaftlichen, politischen und planerischen Diskursen gemeinhin als Problem thematisiert. Das gilt zumindest dann, wenn er von gesellschaftlichen Gruppierungen ausgeübt wird, die nicht in das auf Hochglanz polierte Image deutscher Innenstädte zu passen scheinen. Treffpunkte von Drogen- und Alkoholabhängigen werden daher vielfach als Schandflecken im Stadtbild angesehen. Der vorliegende Beitrag ist diesen Treffpunkten gewidmet. Am Beispiel der dort stattfindenden Praktik des kollektiven Alkoholkonsums untersuche ich, inwiefern auf den ersten Blick banal erscheinende Praktiken wie das gemeinsame Trinken alkoholischer Getränke dazu beitragen, öffentliche Räume zu gestalten.

Vor dem Hintergrund eines ko-konstitutiven Verständnisses von der Gestaltung öffentlicher Räume verschiebt sich die Zusammensetzung der Teilnehmerinnen und Teilnehmer an Planungsprozessen ebenso wie deren Ort und Fokus. Während die Gestaltung öffentlicher Räume klassischerweise als zentrale Aufgabe von Planung auf verschiedenen, institutionell gefassten Maßstabsebenen wie Bund, Land oder Gemeinde angesehen wird, die sich räumlich in den Büros der Verwaltung, den Plenarsälen der Politik oder an den sprichwörtlichen Runden Tischen diverser Beteiligungsverfahren verorten lässt, schlägt der vorliegende Beitrag ein grundlegend anderes Planungsverständnis vor. Unter Rückgriff auf aktuelle Theorieentwicklungen im Bereich raumbezogener Praktikentheorien (für einen Überblick vgl. Schatzki/Knorr Cetina/von Savigny 2001; Nicolini 2012; Schäfer/Everts 2019) werden sowohl die Praktiken der Planerinnen und Planer als auch die Konsumpraktiken der Drogen- und Alkoholabhängigen als Teil eines konstitutiven Wechselspiels aufgefasst, in dem sich aufeinander gerichtete Praktiken ,auf Augenhöhe' begegnen und in Aushandlungsprozesse miteinander treten, aus denen die soziomaterielle Gestaltung öffentlicher Räume als Ergebnis hervorgeht. Der Planungsprozess verlagert sich damit zu einem nicht unwesentlichen Teil an die Treffpunkte der Konsumenten, klassische Zieldefinitionen von Planung werden brüchig und müssen die teils widerständigen Praktiken der Mitglieder der Drogen- und Alkoholkonsumszene integrieren.

Dabei ist der inhaltliche Gegenstand des vorliegenden Beitrags kein neuer. Zahlreiche Artikel haben sich in den vergangenen Jahren mit Drogen- und Alkoholkonsum auseinandergesetzt, auch im Hinblick auf geographische Raumbezüge (vgl. u. a. Jayne/Holloway/Valentine 2006; Jayne/ Valentine/Holloway 2010; Kraehnke 2014; Warf 2014). Gegenüber anderen Ansätzen birgt die Betrachtung von Praktiken jedoch einen zentralen Vorteil, denn vor einem praktikentheoretischen Hintergrund lassen sich wissenschaftliche Debatten aufeinander beziehen, die bisher weitgehend ent- koppelt voneinander stattfinden. So könnte man stark theoretisch orientierten Artikeln aus einer anwendungsorientierten Planungsperspektive heraus vorwerfen, keine Handlungsempfehlungen für die planerische Praxis zu liefern. Umgekehrt kann man Teile der planerischen Praxis als zu theoriearm identifizieren. Diese Lücke können praktikentheoretische Ansätze schließen, indem sie hochgradig theoriegeleitet argumentieren, sich in ihrer Argumentation jedoch immer auf das praktische Handeln der Menschen vor Ort beziehen. Indem ich im Folgenden sowohl die individuellen und kollektiven „doings and sayings“ (Schatzki 1996: 89) der Szenemitglieder als auch den förmlichen stadtplanerischen Umgang mit diesen als Teil eines Wechselspiels von Praktiken begreife, denke ich, diesen zentralen Vorteil für die Planungspraxis fruchtbar machen zu können.

Um dieses Ziel zu erreichen, soll zunächst ein kurzer Überblick über die Grundzüge praktikentheoretischer Denkansätze gegeben werden. Auf diesem Wege arbeite ich das maßgebliche Erkenntnisinteresse heraus, welches diese Ansätze zu einer Gruppe von Praktikentheorien vereint (Kapitel 2). Im nächsten Schritt werden Mechanismen des $\mathrm{Zu}$ sammenwirkens von Praktiken und (sozialen wie materiellen) Ordnungen bestimmt und zu sites of the social als zeiträumlich verortbarem Untersuchungsgegenstand verdichtet (Kapitel 3). Nachdem die für den empirischen Feldzugang angewendeten wissenschaftlichen Forschungspraktiken offengelegt wurden (Kapitel 4), befasst sich der vorliegende Beitrag mit der Frage, wie im Wege von Praktiken des kollektiven Alkoholkonsums sites of the social hervorgebracht werden, die einerseits Ergebnis vorangehender Praktiken sind und andererseits spezifische Folgepraktiken ermöglichen oder begrenzen (Kapitel 5). Dieses Beispiel soll sodann genutzt werden, planungswissenschaftliche Ansätze durch die Einbeziehung von Praktikentheorien auf einer grundlegend theoretischen Ebene weiterzuentwickeln (Kapitel 6). In einem abschließenden Fazit werden die zuvor herausgearbeiteten theoretischen Argumente auf die planerische Praxis übertragen. Damit möchte ich zeigen, wie sehr praktikentheoretische Ansätze, trotz aller Theorieorientierung, die Arbeit der Planerinnen und Planer bereichern können.

\section{Praktikentheorien als theoretischer Zugangspunkt}

In diesem Beitrag spreche ich von Praktikentheorien stets im Plural, denn die eine Praktikentheorie im Sinne eines homogenen, in sich geschlossenen Forschungsansatzes, der nur noch nach dem berühmten Schema F zur Anwendung gebracht werden muss, gibt es nicht. Vielmehr bilden Praktikentheorien eine Art bunten Blumenstrauß lose mitein- 
ander verwandter Theorieansätze, welcher bei einer ersten Annäherung zunächst verwirrend erscheinen kann. Noch dazu entwickelt sich das Feld praktikentheoretischer Ansätze zurzeit mit einer großen Dynamik, insbesondere was die raumwissenschaftliche Rezeption dieser Ansätze angeht (vgl. Everts/Schäfer 2019: 8). Gleichzeitig steht das praktikentheoretische Denken jedoch in einer langen Tradition nicht ohne Weiteres miteinander kompatibler Denkansätze. So verweist Hillebrandt (2009: 369-370) unter anderem auf die im Jahr 1848 erstmals veröffentlichten Feuerbachthesen von Karl Marx ${ }^{1}$ als frühen Versuch, im Begriff der Praxis das theoretische Denken mit der Lebenswirklichkeit der Menschen zu verbinden. Ob man die Feuerbachthesen allein deshalb als einen frühen praktikentheoretischen Ansatz identifizieren kann, mag dahingestellt bleiben. Spätestens jedoch die soziologischen Arbeiten von Pierre Bourdieu oder Anthony Giddens können als Praktikentheorien der ersten Generation bezeichnet werden (vgl. Everts/Schäfer 2019: 9). Während soziale Praxis bei Bourdieu (1987: 98) den „Ort der Dialektik von opus operatum und modus operandi, von objektivierten und einverleibten Ergebnissen der historischen Praxis, von Strukturen und Habitusformen" beschreibt, aus denen heraus sich entlang vielfältiger Distinktionslinien gesellschaftliche Ungleichheit manifestiert, rückt Giddens in seinem Konzept der Dualität der Struktur das produktive Moment von Strukturen in den Vordergrund, indem er darlegt, dass Struktur ,nicht mit Zwang gleichgesetzt werden [darf]: sie schränkt Handeln nicht nur ein, sondern ermöglicht es auch“ (Giddens 1997: 78).

Der vorliegende Beitrag ist ganz wesentlich durch die Arbeiten der sogenannten zweiten Generation von Praktikentheoretikerinnen und -theoretikern geprägt, zu deren wichtigsten Vertretern sich Theodore Schatzki, Andreas Reckwitz sowie Elizabeth Shove, Mika Pantzar und Matt Watson zählen lassen. In der deutschsprachigen Geographie wurde die Diskussion um Praktikentheorien unter anderem durch Jonathan Everts (vgl. Everts/Lahr-Kurten/Watson 2011; Everts 2016) und Matthias Lahr-Kurten (vgl. LahrKurten 2012; Lahr-Kurten 2018) geprägt. Die Potenziale praktikentheoretischer Ansätze für die planungswissenschaftliche Diskussion wurden von Zimmermann (2017)

\footnotetext{
${ }^{1}$ Marx kritisiert darin die von Ludwig Feuerbach (1841) entwickelten materialistischen Ansätze der Religionskritik dafür, dass „der Gegenstand, die Wirklichkeit, Sinnlichkeit, nur unter der Form des Objekts oder der Anschauung gefasst wird; nicht aber als sinnlich menschliche Tätigkeit, Praxis; nicht subjektiv“ (Marx 1845: o.S.). Dem hält Marx in der achten Feuerbachthese entgegen: „Alles gesellschaftliche Leben ist wesentlich praktisch. Alle Mysterien, welche die Theorie zum Mystizismus veranlassen, finden ihre rationelle Lösung in der menschlichen Praxis und im Begreifen dieser Praxis“ (Marx 1845: o.S.).
}

mit durchaus positivem Ergebnis ausgelotet. Was jedoch eint diese Ansätze und lässt sie für raumwissenschaftliche Analysen so interessant erscheinen?

Praktikentheoretische Ansätze der zweiten Generation gehen davon aus, dass gesellschaftliche Zusammenhänge und deren Wandlungsprozesse weder durch vorgefundene Strukturen noch durch individuelles Handeln allein erklärt werden können. Sie sind daher wesentlich durch ihren Anspruch geprägt, den wissenschaftstheoretischen Graben zwischen individualistischen und strukturalistischen Sichtweisen zu überwinden, ohne dabei einer der beiden Seiten Priorität einzuräumen (vgl. Reckwitz 2003: 287; Schatzki 2003: 181; Alkemeyer/Buschmann 2016: 116-118; Hillebrandt 2016: 73). Praktisches Handeln ist dabei immer auch materielles Handeln in körperlichen Gesten und Tätigkeiten, an physischen Orten und mit konkreten Gegenständen (Reckwitz 2017: 114). Im Gegensatz zu individualistischen Ansätzen ist dieses Handeln jedoch kein singulär zu betrachtender Akt, der im Hier und Jetzt dem freien, unbeeinflussten Willen der Handelnden entspringt, sondern Teil eines offenen, räumlich-zeitlich verteilten Zusammenhangs von Taten und Worten (,open-ended, spatially-temporally dispersed nexus of doings and sayings"; Schatzki 2012: 14), welcher als Praktik bezeichnet wird und neben der Aktivitätskomponente des praktischen Handelns auch strukturelle Organisationskomponenten in sich trägt (vgl. Schatzki 2002: 77; Lahr-Kurten 2012: 50-51; Schatzki 2017: 130). Weder Struktur noch Handlung sind also der Praxis vorgängig. Vielmehr bilden Praktiken, die sowohl eine Strukturals auch eine Handlungsdimension beinhalten, den ontologischen Ausgangspunkt der sozialen wie auch der materiellen Welt. Gemäß meiner einleitenden Feststellung, dass es die eine Praktikentheorie nicht gibt, bediene ich mich im Folgenden daher praktikentheoretischer Argumente aus unterschiedlichen Diskussionssträngen und nutze diese für eine theoretische Anreicherung, vielleicht sogar für eine Weiterentwicklung der planungswissenschaftlichen Diskussion.

\section{Verdichtung von Praktiken und Ordnungen in „sites of the social“}

In praktikentheoretischen Ansätzen sind Praktiken und (materielle wie soziale) Ordnungen untrennbar miteinander verwoben. Sie ermöglichen und beschränken einander (vgl. Schatzki 2002: 117; Lahr-Kurten 2012: 56-58). Aufgrund der körperlichen Artikulation praktischen Handelns, das sich in der einen oder anderen Form immer auf seine Umgebung richtet, können Praktiken niemals losgelöst von den mit ihnen in Zusammenhang stehenden materiellen Arrangements betrachtet werden (vgl. Pink 2012: 3; Schatzki 2019: 58). Materielle Arrangements bilden da- 
mit einerseits den Kontext, durch den das Stattfinden von Praktiken überhaupt erst interpretier- und verstehbar wird, andererseits wird dieser Kontext selbst erst vor dem Hintergrund der dort stattfindenden Praktiken mit Sinn versehen (vgl. Everts/Lahr-Kurten/Watson 2011: 329-330; Shove/ Pantzar/Watson 2012: 130-134; Dünckmann/Fladvad 2016: 28). Diejenigen Orte und Momente, an und in denen sich materielle Arrangements und (individuelle wie kollektiv geteilte) Praktiken zu soziomateriellen Ordnungen verbinden, die gleichermaßen durch menschliches Handeln strukturiert sind als auch dieses strukturieren, werden als sites of the social bezeichnet (Schatzki 2002: 138; Schmid/ Reda/Kraehnke et al. 2019: 99). Mit dem Fokus auf diese räumlich und zeitlich definierten Situationen und die in diesen stattfindenden körperlichen Praktiken sowie die darin manifestierten Bedeutungen gestatten es Praktikentheorien, komplexe (sub)gesellschaftliche Zusammenhänge einer sozialtheoretisch fundierten raumbezogenen Analyse zugänglich zu machen und zugleich im Sinne einer holistischen Perspektive „den Blick zu weiten und eine neue Sensibilität zu entwickeln, um die Heterogenität und Widersprüchlichkeit des Sozialen zu begreifen“ (Reckwitz 2016: 11).

\section{Empirische Feldzugänge}

Die Gleichzeitigkeit von theoretischer Strenge und empirischer Offenheit soll im Folgenden genutzt werden, um die Treffpunkte einer offenen Drogen- und Alkoholkonsumszene im Kieler Stadtteil Gaarden aus einer praktikentheoretisch informierten Perspektive zu betrachten. Dazu wurden, ganz im Sinne dieser Offenheit, drei Formen des Feldzugangs miteinander verschnitten:

Während einer mehrmonatigen Hospitation als Streetworker in der aufsuchenden Sozialarbeit war ich regelmäßig an diesen Orten präsent und konnte die dort stattfindenden Praktiken aus erster Hand beobachten, zuweilen sogar Teil dieser Praktiken sein. Diese Erfahrungen bilden das Kontextwissen, vor dessen Hintergrund die Ergebnisse der anderen Methoden interpretiert und mit denen sie immer wieder abgeglichen werden müssen. Mehr noch: Durch meine mehrmonatige aktive Teilnahme an den Interaktionen und Praktiken im Feld war es mir - zumindest zeitweise möglich, am Alltagsleben der Beforschten zu partizipieren. Die durchgeführte Untersuchung trägt daher durchaus ethnographische Züge. Gegenüber stärker standardisierten Forschungsmethoden bietet dies im vorliegenden Fall eine Reihe von Vorteilen:

Durch die anhaltende Präsenz von Beobachtenden am Ort des Geschehens erfolgt eine zeitliche Streckung der Erhebungsphase, woraus sich anstatt einer nur punktuellen
Informationsaufnahme deutlich vielfältigere Gelegenheiten zur Aufnahme ebenso vielfältiger Informationen ergeben können, die eine „dichte Beschreibung“ der „Vielfalt komplexer, oft übereinandergelagerter oder ineinander verwobener Vorstellungsstrukturen“ (Geertz 1983: 15) überhaupt erst möglich machen. Infolge der längeren Verweildauer im Feld erlaubt ein ethnographisches Vorgehen zudem eine nicht unerhebliche Verschiebung der Perspektive. Statt feste Strukturen des Feldes zu ergründen, gerät die prozessuale Dimension des menschlichen Lebens, mithin seine Kontingenz, in den Fokus des Forschungsinteresses (vgl. Hammersley/Atkinson 2007: 234). In einem solchen Sinne kann die Ethnographie eine wichtige gesellschaftliche Funktion als Korrektiv gegenüber Alltagswissensbeständen sowie gegenüber den Wissensbeständen nomothetisch operierender Forschungstraditionen übernehmen (Hammersley/Atkinson 2007: 235).

Als ,opportunistische und feldspezifische Erkenntnisstrategie“ sind Ethnographien ,nicht regulative, sondern mimetische Formen empirischer Sozialforschung" (Amann/ Hirschauer 1997: 20). Ein gewisser Kontrollverlust über die Bedingungen des Erkenntnisprozesses führt damit nicht zwangsläufig zum Scheitern des Projekts. Vielmehr wird er ,zu einer methodisch notwendigen Freiheit für den Forschungsprozess" (Ammann/Hirschauer 1997: 17), die dazu beiträgt, eine zu dominante A-priori-Vorstrukturierung des Erkenntnisgegenstandes zu vermeiden und so überhaupt erst die Voraussetzung für ein ,thinking outside the box“ schafft. Gegenüber reinen Interviewverfahren hat die Ethnographie als synchrone Beobachtung lokaler Praxis außerdem den Vorzug ,eben nicht nur die Selbstbeschreibungen, d. h. die Interpretationen, Meinungen und kognitiven Wissensbestände der Teilnehmer zu erheben, sondern die (präreflexiven) ,Selbstformulierungen“ ihrer Praxis“" (Ammann/Hirschauer 1997: 23-24) zu erschließen. Dadurch kann internalisiertes Wissen freigelegt werden, das auf bloße Nachfrage hin nicht verfügbar wäre, weil es von den Teilnehmenden im Bereich des Selbstverständlichen abgelegt und zeitgleich aus dem Bereich reflexiven Wissens entfernt worden ist.

Ergänzt wurde das grundlegend ethnographische Vorgehen durch ein partizipatives Fotoprojekt mit denjenigen, die einen großen Teil ihres Alltags an den untersuchten Szenetreffpunkten verbringen: den Mitgliedern der Drogen- und Alkoholkonsumszene von Kiel-Gaarden selbst. Im Rahmen des Fotoprojekts haben einige der Szenemitglieder ihren ganz persönlichen Blick auf die Treffpunkte in Fotos festgehalten (für eine allgemeine Befassung mit fotografischen Methoden als Erhebungsinstrumente der empirischen Sozialwissenschaften vgl. u. a. Collier/Collier 1986; Harper 1988; Rose 2016 sowie für die reflexive Fotografie als Methode der empirischen Humangeographie Dirksmeier 2009; Dirksmeier 2013). Unter dem Slogan „Zeig mir Dein Gaar- 
den!“ wurden die Szenemitglieder aufgefordert, eine Woche lang fotografisch zu dokumentieren, was diesen Stadtteil für sie ausmacht. So entstanden rund 150 Fotos von sechs Fotografinnen und Fotografen, welche zu einem sehr großen Teil die Szenetreffpunkte zum Inhalt haben. Diese Bilder allein offenbaren die Vielfalt der dort stattfindenden Praktiken und wurden daher einer ersten bildanalytischen Interpretation in Anlehnung an Niesyto (2006) unterzogen.

Zuletzt wurden die entstandenen Fotografien als Einstieg in qualitative Interviews mit den Fotografinnen und Fotografen selbst genutzt, um die hinter dem beobachtbaren praktischen Handeln verborgen liegenden Organisationskomponenten von Praktiken herauszufiltern. Auf die Anwendung eines Leitfadens wurde dabei ganz bewusst verzichtet, um dem Gesprächsverlauf nicht von vornherein allzu starre Leitplanken zu setzen und stattdessen den Gesprächspartnerinnen und -partnern zu ermöglichen, ihre eigenen Deutungsmuster und Relevanzsysteme zu entfalten (vgl. Helfferich 2009: 114). Außerdem wurde viel Wert auf die „Schaffung einer möglichst natürlichen Kommunikationssituation in der Alltagswelt der Beforschten“" (Rothfuß/Dörfler 2013: 25) gelegt. Daher wurden alle Interviews an Orten geführt, die den Beteiligten aus ihrem Alltag bereits vertraut waren: Sitzend auf einem Brunnen, der im Sozialraum des Stadtteils als wichtiger Szenetreffpunkt in Erscheinung tritt, auf einer Parkbank im Grünen oder in einer Gartenhütte, in der einige der Szenemitglieder sich regelmäßig aufhalten. Auf diese Weise entwickelten sich natürliche Gespräche auf Augenhöhe, in denen die Interpretation der Fotos und der auf ihnen zu erkennenden Praktiken vornehmlich durch die Fotografinnen und Fotografen selbst vorgenommen wurde. Meine eigene Interpretation trat demgegenüber in den Hintergrund, fungierte jedoch als Reservoir für die Generierung von Nachfragen und zur Einordnung des Gesagten. Sämtliche Interviews wurden vollumfänglich transkribiert. Auf eine Notation unter Berücksichtigung von Sprechpausen, Stimmlagen, Dialekten und sonstigen nonverbalen oder parasprachlichen Elementen wurde hingegen weitestgehend verzichtet, da ein solches Verfahren für die durchgeführte Untersuchung nur wenig zusätzlichen Erkenntnisgewinn versprach und zudem die flüssige Lesbarkeit der Transkripte erschwert. $^{2}$

Die eigenen ethnographischen Beobachtungen, sämtliche Bilder mitsamt der an ihnen durchgeführten bildanalytischen Interpretationen sowie die Transkriptionen der auf dieser Ausgangsbasis geführten Interviews bilden den

\footnotetext{
2 Zum Für und Wider aufwendiger Notationssysteme in qualitativen Interviewverfahren vgl. jedoch Gläser/Laudel (2006: 188), Meuser/Nagel (2002: 83) sowie Mattissek/Pfaffenbach/Reuber (2013: 191-196).
}

Datenkorpus und damit die „,neue Realität“ (Flick 2007: 384) der weiteren Analyse. Dieser Datenkorpus wurde entlang des a posteriori aus dem Zusammenspiel von Theorie, ethnographischen Beobachtungen und den in Bildern und Interviews aufscheinenden Deutungshorizonten der Szenemitglieder entwickelten Kategoriensystems neu strukturiert. Dabei hat sich die im weiteren Verlauf für diesen Beitrag zu untersuchende Praktik des gemeinsamen Alkoholkonsums als besonders relevantes Element der Lebenswelt der Szenemitglieder herausgestellt. Dieses präreflexiv stattfindende und konkret beobachtbare Element steht daher im Zentrum meiner folgenden Überlegungen und wird, wo es zielführend erscheint, mit Auszügen aus den geführten Interviews oder Hinweisen auf die entstandenen Fotografien angereichert.

Die gewählten Feldzugänge und die in ihnen zur Anwendung kommenden empirischen Forschungsmethoden stellen ihrerseits Praktiken dar. In der Gesamtschau verdichten sich die daraus resultierenden empirischen Ergebnisse also zu einem Bild, das aus dem Zusammentreffen wissenschaftlicher Forschungspraktiken mit den Alltagspraktiken der Beforschten ko-konstitutiv hervorgeht und so eine sehr lebendige, durch die intensive Mitwirkung der Szenemitglieder zuweilen gar emotional geprägte Vorstellung davon vermittelt, wie öffentliche Räume durch raumbezogene Praktiken als Treffpunkte einer Drogen- und Alkoholkonsumszene angeeignet und sozial-räumlich gestaltet werden.

\section{Treffpunkte von Drogen- und Alkoholabhängigen als „sites of the social“"}

Den räumlichen Kontext für die empirische Untersuchung von Treffpunkten der Drogen- und Alkoholkonsumszene bildet der Kieler Stadtteil Gaarden, der sich auf der östlichen Seite der Kieler Förde befindet. Als traditionelles Wohnquartier der Werftarbeiterinnen und Werftarbeiter ist Gaarden in besonderem Maße davon betroffen, dass die Kieler Großwerften seit Jahrzehnten Personal abbauen. Insgesamt sind viele tausend Stellen weggefallen. Wirtschaftszweige, die diesen Stellenabbau auffangen könnten, sind in Gaarden nicht geschaffen worden. Gaarden muss daher als klassischer Verlierer des wirtschaftlichen Strukturwandels bezeichnet werden. Gleichzeitig hat sich die Westseite der Kieler Förde, auf der sich die Innenstadt und der Hauptbahnhof befinden, positiver entwickelt. Mit dem Sophienhof ist in unmittelbarer Nähe des Hauptbahnhofs ein gut frequentiertes Einkaufszentrum entstanden. Rund um den Hauptbahnhof wurden verstärkt Polizeikontrollen vorgenommen, die sich insbesondere auf die dortige Drogenund Alkoholkonsumszene konzentrierten und dazu führten, 


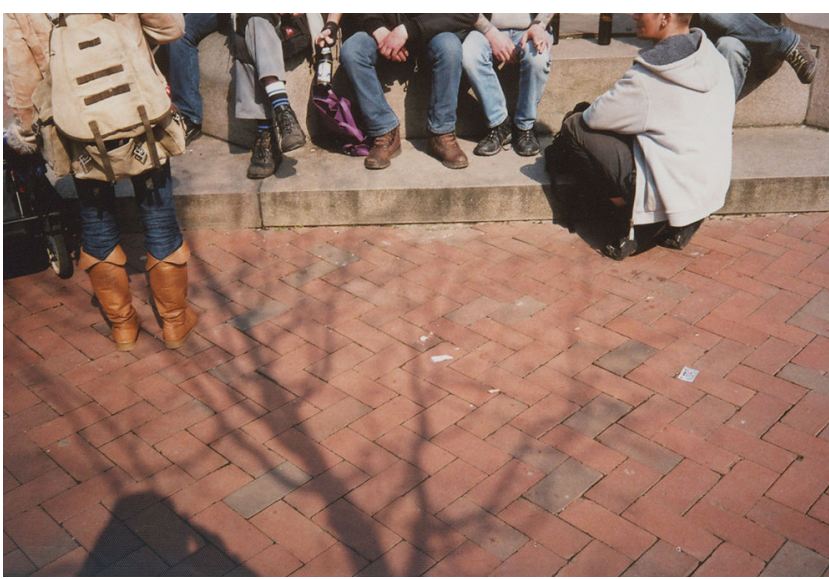

Abbildung 1 Eine Gruppe von Szenemitgliedern am Szenetreffpunkt Vinetaplatz (Fotograf: Achim)

dass das Bahnhofsumfeld für die Szenemitglieder immer unattraktiver wurde. Schließlich kam es zu einer weitgehenden Verdrängung der Szene aus dem Bahnhofsumfeld. Eine Koalition aus wirtschaftlichen Interessen, stadtplanerischen Praktiken, wie der Planung und Genehmigung eines Einkaufszentrums, und staatlichen Kontrollpraktiken hatte gegenüber den lebensweltlichen Praktiken der Szenemitglieder die Oberhand behalten. Im Stadtteil Gaarden hingegen waren die staatlichen Kontrollorgane weniger präsent. Konsumenten bekamen bessere Chancen, ihre Praktiken auszuleben, da diese hier weniger häufig in einen Wettstreit mit den Planungs- und Kontrollpraktiken der öffentlichen Instanzen treten mussten. In der Folge kam es zu einer Verlagerung von Teilen der Drogen- und Alkoholszene vom Hauptbahnhof nach Gaarden, wo sich Szenetreffpunkte bildeten, die bis heute im Bild des Stadtteils präsent sind.

Im Folgenden werde ich einen praktikentheoretischen Zugang als „Aussichtsplattform“ (Schmid/Reda/Kraehnke et al. 2019: 100) nutzen, um die Treffpunkte der Drogenund Alkoholabhängigen von Kiel-Gaarden in Augenschein zu nehmen. Eine auf die qualitative Erfassung aller an diesen Orten sichtbaren Praktiken gerichtete Untersuchung würde jedoch Gefahr laufen, ,eine unendliche Reihe von Episoden detailliert nachzuerzählen, ohne daraus allgemeinere Theoriebausteine bilden zu können" (Zimmermann 2017: 16). Daher werde ich mich an dieser Stelle auf die beispielhafte Beschreibung einer einzigen der zahlreich zu beobachtenden Praktiken beschränken: derjenigen des gemeinsamen Trinkens. Anhand dieses Beispiels möchte ich exemplarisch aufzeigen, wie durch praktisches Handeln und die dahinterliegenden Organisationskomponenten - kurz: durch Praktiken - materielle Arrangements so (um)genutzt werden, dass sie aus der Perspektive der Szenemitglieder

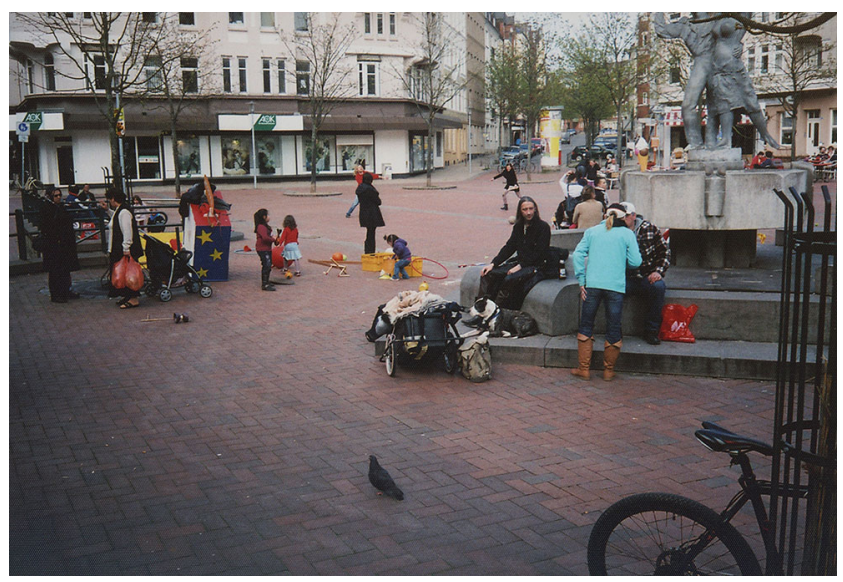

Abbildung 2 Szenemitglieder auf den Stufen des Brunnens am Vinetaplatz (Fotograf: Achim)

als Treffpunkte - oder theoretischer formuliert: als sites of the social - fungieren.

Praktiken des gemeinsamen Trinkens der Szenemitglieder werden in den Fotos und den darauf aufbauenden Interviews verhältnismäßig häufig adressiert (vgl. Abbildungen 1 und 2). Beide Aufnahmen sind zu unterschiedlichen Zeitpunkten entstanden und zeigen eine - zumindest in Teilen - unterschiedliche Personenkonstellation. Trotz ihrer Unterschiedlichkeit offenbaren die Aufnahmen jedoch eine Reihe von Faktoren, die einerseits typisch für die Konsumpraktiken der untersuchten Szene sind und diese andererseits von denjenigen Praktiken unterscheiden, mit denen der Konsum alkoholischer Getränke in großen Teilen der bürgerlichen Gesellschaft verbunden ist. Unter anderem zeigt sich dies am Gegenstand des Konsums. Dem Etikett und der Flaschenform nach wird in beiden Situationen Bier der Marke Oettinger getrunken. In der erkennbaren 0,5-LiterFlasche handelt es sich dabei um eines der günstigsten am Markt erhältlichen Biere. Dass die Konsumpraktiken der Drogen- und Alkoholkonsumszene fast ausschließlich dieses Bier zum Gegenstand haben, symbolisiert ein eher niedriges sozioökonomisches Niveau. Das wäre für sich genommen wenig überraschend. Zugleich wird jedoch im Wege des kollektiven Konsums eines einheitlichen Gutes - mithin im Wege einer Praktik - Gemeinschaft hergestellt. Ob es sich dabei um eine bewusste Entscheidung handelt, ist unerheblich, da sich der Konsum des ,einheitlichen Gutes Oettinger" bereits als Markenzeichen der Szene etabliert hat. Auch der Ort des Geschehens erscheint aufschlussreich und wäre für bürgerliche Teile der Gesellschaft ein eher atypischer Raum für den Konsum alkoholischer Getränke. Während wir Alkoholkonsum in der Regel im Schutz der eigenen vier Wände oder in eigens zu diesem Zweck hergerichteten Lokalitäten praktizieren, findet er hier auf den 
Stufen eines Brunnens im öffentlichen Raum statt, ohne Tische, ohne Stühle, ohne Gläser und ohne die freundliche Bedienung, die auf Zuruf den Nachschub garantiert. Dennoch wird dieser Raum von den Szenemitgliedern fast täglich frequentiert und dabei stets für die gleichen Praktiken genutzt, unter anderem für das gemeinsame Trinken, wie ein Szenemitglied im Interview betont: „Also ich bin ja auch auf der Szene ... Ich hätte keine Lust irgendwie fünfzehn Leute bei mir in die Wohnung zu holen. Dann trifft man sich doch lieber irgendwo, kann gehen, kann kommen, man trinkt Bier zusammen, man unterhält sich und geht irgendwann wieder auseinander so" (Interview Rolf). Obwohl beide Aufnahmen Gruppen zeigen, die einander zugewandt sind, bedingen die verfügbaren materiellen Arrangements andere Formen des gemeinschaftlichen Trinkens, als sie in großen Teilen der bürgerlichen Gesellschaft üblich wären. Ein Gegenübersitzen ist aufgrund der räumlichen Konstellation nur schwer möglich. Es wird vorwiegend nebeneinander gesessen oder gar gestanden. Der eigentliche Akt des Konsums wird nicht durch Anstoßen oder Zuprosten zelebriert, sondern erfolgt selbstverständlicher und ohne große Gesten. Gläser werden nicht verwendet, der Alkoholkonsum ist durch einen pragmatischen Zugang geprägt. Er scheint eher dem täglichen Leben und Überleben auf der Straße zu dienen als dem bewussten Genießen eines besonderen Moments.

Durch das spezifische ,was?“", ,wann?“, ,,wo?“, ,,warum?“ und ,wie?“ der zu beobachtenden Praktik werden Menschen, Bierflaschen und Sitzgelegenheiten, in einem weiteren Sinne aber auch Transportbehältnisse wie Rucksäcke oder Anhänger und Begleitfaktoren wie Zigaretten et cetera als Teil von Praktiken-Arrangement-Bündeln in einen inhaltlichen Zusammenhang zueinander gestellt, der durch die Praktik des gemeinschaftlichen Alkoholkonsums zusammengehalten wird. Gleichzeitig befindet sich dieses Praktiken-Arrangement-Bündel an einem konkreten Ort in der realen Welt, an dem es den Raum als Ort des Stattfindens dieser Praktiken entscheidend prägt. So schafft die gemeinschaftlich geteilte Praktik des Trinkens einerseits die genannte Einheit zwischen den Szenemitgliedern und wirkt nach innen insofern als Einhegungs- und Inklusionsmotor, der zugleich an andere Inklusionsmechanismen wie gemeinsames Rauchen, Sich-Unterhalten oder gegenseitige Hilfe in Notsituationen anschließt. Ein Szenemitglied formuliert das wie folgt: „Das geht auch um die Ehre oder so. Und wir halten dann eben auch ... das, was uns noch geblieben ist, da halten wir dann eben auch zusammen oder so. Oder man hilft sich aus mit Kohle oder mit Essen oder mit Klopapier oder mit allem. Und wenn einer was über hat oder nicht mehr braucht oder so, dann wird das erst mal unter Kollegen angeboten. Also das ist schon so eine soziale Gemeinschaft, ja. Wo auch immer alle Leute wiederkommen“ (Interview Achim). Auf meine Rückfrage, ob es dann nicht egal sei, wo man sich treffe, stellt Achim selbst den Bezug zur Praktik des kollektiven Alkoholkonsums her: „Naja, du musst schon in der Nähe einer Lokalität sein, wo du was zu trinken kaufen kannst ..." (Interview Achim). Nach außen, das heißt gegenüber denjenigen, die nicht Teil der Praktikengemeinschaft (vgl. hierzu auch den von Lave und Wenger (1991) sowie Wenger (1998) am Beispiel des gemeinschaftlichen Lernens entwickelten „Communities of Practice“-Ansatz) sind, fungiert das gemeinschaftliche Trinken hingegen als Abgrenzungsmechanismus. Ein Ort der regelmäßig von Drogen- und Alkoholabhängigen frequentiert und zum gemeinsamen Konsum genutzt wird, lädt eben selten andere dazu ein, dort Praktiken wie ein sonntägliches Familienpicknick oder eine geschäftliche Videokonferenz abzuhalten. Ein Szenemitglied berichtet dementsprechend über das Zusammentreffen mit dem Inhaber der lokalen Eisdiele: „Und da jetzt der Winter vorbei ist, hat er uns vor zwei, drei Tagen hat er uns da jetzt weggeschickt. Ich meine hätten wir eine Cola in der Hand gehabt, wäre das wohl kein Thema gewesen, aber da ging es wohl um das Bier. Kann ich auch ein bisschen verstehen, muss ich sagen, weil es waren viele Kinder da, die da Ball gespielt hatten" (Interview Karsten). Weiter führt er aus: „Es gibt keinen optimalen Ort für solche Leute. Es sei denn man schafft einen. Und optimaler Ort wäre: genügend Sitzmöglichkeiten, vielleicht eine Grillmöglichkeit für den Sommer, eine Getränkemöglichkeit, aber nicht nur ... okay, Getränke mitbringen ist ja schon mal nicht schlecht, aber cooler wäre noch am besten gleich noch ein Shop nebenan. Und ... sowas müsste man haben. Eine Toilette müsste man haben. Und man müsste den Leuten das so präsentieren, dass sie das Gefühl haben, auch was wert zu sein. Und nicht der Abschaum zu sein. Dadurch, dass man den Leuten nichts gibt, müssen sie sich ja irgendwo was nehmen. Und die kommen aus $\mathrm{Sky}^{3}$ raus, zack, Bier auf, ja hier setze ich mich hin, weil da habe ich gerade den getroffen, schwuppdiwuppdiwupp ist eine neue Szene gebildet“ (Interview Karsten). Als regelmäßiges Aktivitätsmuster ist das Trinken von Alkohol an Treffpunkten im öffentlichen Raum daher zugleich Teil alltäglicher Gewohnheiten und Routinen der Szenemitglieder, in deren beständiger Wiederholung Formen alltäglicher Räumlichkeit produziert und reproduziert werden (vgl. Shove/Pantzar 2016: 109; Reda/Runkel 2019: 206), als auch Bestandteil einer weiter gefassten Ebene von Praktiken als „gesellschaftlich konventionalisierte und angeordnete $\mathrm{Zu}$ sammenhänge des Tuns und Sagens" (Geiselhart/Winkler/ Dünckmann 2019: 27 unter Bezugnahme auf Schatzki 2002). Als solche sind sie typisch für den Treffpunkt,

\footnotetext{
3 ein Lebensmittelmarkt.
} 
unterscheiden sich aber von außerhalb des Treffpunkts akzeptablen Verhaltensweisen zuweilen recht deutlich und stehen gelegentlich sogar mit diesen im Wettbewerb um Zeit und Raum (vgl. Shove/Pantzar/Watson 2012: 78-79; Jackson/Brembeck/Everts et al. 2018: 118-119; Everts/ Geiselhart/Rominger et al. 2019: 229-231). Im Zuge von Praktiken des gemeinschaftlichen Trinkens artikulieren sich demnach „die feinen Unterschiede“ (Bourdieu 1982), über welche die Distinktionslinien zwischen denjenigen, die zur Praktikengemeinschaft gehören, und denjenigen, für die dies nicht gilt, nicht nur erfahrbar, sondern überhaupt erst hervorgebracht werden. Oder anders ausgedrückt: Es existiert keine natürliche Grenze entlang sozialer oder ökonomischer Kategorien, die den Treffpunkt als site of the social einer bestimmten Gruppe definiert. Vielmehr erfolgt eine solche Definition im Wege von Inklusions- und Exklusionsmechanismen, die einer raumbezogenen „Logik der Praxis“ (Hörning/Reuter 2004: 14) folgen, welche ihrerseits das Sag- und Machbare - also weitere Praktiken ermöglicht und begrenzt (vgl. Gherardi (2017: 49) sowie die grundlegenden Überlegungen von Giddens (1997) zur Dualität der Struktur). In Abgrenzung zu Dünckmann und Fladvad (2016: 27, 44-45) beinhalten Praktiken nach meinem Verständnis daher immer ein politisches Moment, selbst dann, wenn sie so banal sind wie das gemeinsame Konsumieren alkoholischer Getränke.

\section{Praktiken der Aneignung öffentlicher Räume als Teil von Planungsprozessen}

Raumbezogene Grenzziehungen sind, wie im voranstehenden Kapitel am kollektiven Alkoholkonsum von Mitgliedern der Drogen- und Alkoholkonsumszene beispielhaft dargelegt wurde, sowohl Ergebnis von Praktiken als auch Voraussetzung für deren Stattfinden. Zugleich stehen diese Praktiken hinsichtlich Zeit und Raum im Wettbewerb miteinander. Daraus folgt, dass Praktiken hinsichtlich räumlicher Grenzziehungen in dreifacher Weise in Planungsprozesse eingewoben sind:

- Der zu beplanende Raum ist als soziomaterielles Arrangement stets schon das Ergebnis von vorangehenden Planungsprozessen. Raumbezogene Planungspraktiken haben demnach den zu beplanenden Raum hervorgebracht.

- Der zu beplanende Raum bildet mit seinen spezifischen Eigenheiten und Problemen den Anlass und die Ausgangsbasis für planerische Veränderungen. Als soziomaterielles Arrangement bringt er demnach raumbezogene Planungspraktiken hervor.
- Räumliche Nutzungen, zum Beispiel in Form gemeinschaftlichen Trinkens, sind aus praktikentheoretischer Perspektive konstitutiver Bestandteil soziomaterieller Arrangements. Diese wiederum stehen in einem konstitutiven Wechselverhältnis zu Planungsprozessen (vgl. die ersten beiden Punkte) und in Konkurrenz zu alternativen Nutzungsmöglichkeiten.

Vor dem Hintergrund dieser Wechselbeziehungen zeigt sich deutlich die Notwendigkeit, Planung aus praktikentheoretischer Perspektive grundsätzlich neu zu denken und herauszufinden, was dies im spezifischen Fall für den planerischen Umgang mit Treffpunkten der Drogen- und Alkoholkonsumszene bedeutet.

Klassischerweise obliegt die Planungshoheit der öffentlichen Verwaltung, die ihren Planungsauftrag durch Anwendung von Raumordnungsgesetz, Baugesetzbuch und anderen Rechtsgrundlagen im Wechselspiel mit den zuständigen kommunalpolitischen Gremien wahrnimmt (Langhagen-Rohrbach 2010: 6, 10) und dafür auf etablierte planungstheoretische Grundlagen zurückgreift (vgl. LeGates 2005: 481). Unter Planung kann nach Fürst (2001: 11) demnach „ein systematisches Vorgehen zur Entwicklung von Handlungszielen und -abfolgen über einen längeren Zeitraum“ verstanden werden. Ein derartiger ,normativer Impuls der geplanten Stadtgestaltung“ (Basten/Gerhard 2016: 134) kann unter anderem an der Existenz zahlreicher städtebaulicher Leitbilder und deren Wandel im Laufe der Zeit abgelesen werden, wie beispielsweise der Wandel von der autogerechten Stadt der Nachkriegszeit, die einmal zur Stadt der kurzen Wege werden soll, eindrucksvoll verdeutlicht.

Bis in die 1970er-Jahre waren Planungsprozesse ganz wesentlich durch ein top-down Planungsverständnis geprägt, in dem die staatliche und kommunale Planungsebene den jeweiligen Rahmen für vorzunehmende Planungen vorgab. Die praktische Arbeit der Planerinnen und Planer fügte sich in diesen Rahmen ein, ihre Ergebnisse beeinflussten das tägliche Leben der Bürgerinnen und Bürger. Im Rahmen der mit einem solchen Planungsverständnis einhergehenden Entscheidungsfindungsprozesse, welche sich in konkreten Praktiken wie dem Erstellen von Plänen oder dem Verfassen von politischen Vorlagen ausdrücken, fungieren die in der Regel verwaltungsseitig angestellten Planerinnen und Planer daher als Sachkundige (Lapintie 2010: 604).

In der Praxis lässt sich eine solche lineare Planungslogik jedoch weder in inhaltlicher noch in hierarchischer Hinsicht durchhalten. Vielmehr verlaufen Planungsprozesse inkrementell und iterativ, das heißt, sie erfolgen in kleinen Schritten und sind rückgekoppelt an frühere Festlegungen, widerstreitende Interessen und sich kontinuierlich verändernde Umweltbedingungen (Fürst 2018: 1713; Hamedinger 2018: 990-991). Faktisch wird Planung damit ,zu einem 
Suchprozess nach neuen, konsensfähigen und zukunftsfähigen Lösungen“ (Fürst 2018: 1713). Seit Ende der 1970erJahre haben sich daher alternative Planungsmodelle durchgesetzt, die weniger auf hoheitlich-administrative und stärker auf dialogorientierte Planungsverfahren und Verhandlungslösungen setzen und deren Hervortreten daher auch als „,communicative turn in planning theory“ (Healey 1992: 143; Healey 1996: 217) bezeichnet werden kann. In der Folge kam es zu einem steigenden Stellenwert von sogenannten weichen Steuerungsinstrumenten, die sich unter dem Begriff der (Urban) Governance zusammenfassen lassen (Basten/Gerhard 2016: 135-136).

Wenngleich in Teilen der planungstheoretischen Literatur zwischenzeitlich von einer Rückbesinnung auf die Notwendigkeit einer stärker eingreifenden, strategisch orientierten Planung die Rede ist (vgl. Wiechmann 2018: 1778), so kann dennoch davon gesprochen werden, dass Planung im Verlauf der letzten 50 Jahre partizipativer geworden ist. Dies zeigt sich unter anderem an der Entwicklung einer Vielzahl planerischer Instrumente zur Information und Beteiligung von sowie zur Kooperation mit Bürgerinnen und Bürgern, die von Planungen der Verwaltung betroffen sind. Unter anderem können hier genannt werden (für eine systematischere und umfassendere Darstellung vgl. Bischoff/ Selle/Sinning 2020): Bürger- und Einwohnerversammlungen, Anhörungen und Erörterungen, Beiräte, Ausschüsse und Arbeitsgruppen, Workshops und Foren, Runde Tische, Zukunftswerkstätten und -konferenzen sowie Formate zur digitalen Beteiligung, z. B. partizipative geographische Informationssysteme.

Vielfach werden diese Instrumente um eine Strategie zur medienübergreifenden Ansprache der Betroffenen ergänzt. Durch die zielgerichtete Nutzung von klassischen Printmedien, Websites und sozialen Netzwerken wie Facebook, Twitter oder Instagram sollen möglichst viele Menschen erreicht werden. Dahinter steht das Bestreben, eine möglichst breite Basis von Interessenten für den jeweiligen Prozess zu gewinnen. Doch ist Planung durch mehr Partizipation tatsächlich auch demokratischer geworden?

Zwar hat sich das vorherrschende Planungsverständnis durch die Governance-Diskurse der vergangenen Jahrzehnte (vgl. Rhodes 1996; Stoker 1998; Bache/Flinders 2004) spürbar in Richtung partizipativ orientierter Ansätze verschoben, dennoch wird die Übersetzung dieser Ansätze in konkrete Beteiligungsprozesse vor allem auf der formal-administrativen Ebene organisiert und strukturiert. Von den Planerinnen und Planern wird weiterhin erwartet, dass sie diese Beteiligungsprozesse „konstruktiv steuern, indem sie dafür sorgen, dass die Prozesse strategisch angelegt werden: auf gemeinsame Ziele hin ausgerichtet, an den Stärken und Schwächen einer Region orientiert, kreativ hinsichtlich Zukunftsoptionen, effektiv hinsichtlich des Prozessmanage- ments und inhaltlich orientiert durch planerische Vorgaben“ (Fürst 2018: 1715). Auch im kommunikativen Planungsparadigma bleibt das Handeln der eigentlich Betroffenen daher vielfach ein Teil hierarchisch organisierter Planungsprozesse mit umstrittener Wirksamkeit (Mattissek/Prossek 2013: 208-209). In vielen Fällen werden die Ausgrenzung schwächerer Akteure und die Wirksamkeit sozialer Ungleichheiten auf den Entscheidungsprozess dadurch sogar verschärft (Rosol/Dzudzek 2020: 331). Dies gilt umso mehr, da bildungsferne Schichten, migrantische Milieus und Menschen in prekären Lebenssituationen in vielen Beteiligungsverfahren systematisch unterrepräsentiert sind und diese Verfahren daher hochgradig selektiv wirken (Selle 2011: 7). Daran ändert auch die mittlerweile zu beobachtende Omnipräsenz digitaler Formate nur wenig. Partizipative Ansätze und Bürgerbeteiligung haben das Spektrum planerischer Praktiken demnach zwar erweitert und in Teilen aus den Amtsstuben an die sprichwörtlichen Runden Tische verlagert, dennoch bleiben die Möglichkeiten, sich wirksam in Planungsprozesse einzubringen, einem Teil der Bürgerinnen und Bürger mehr oder weniger verschlossen. Es ist daher nicht zu weit gegriffen, den allermeisten Beteiligungsverfahren ein gewisses demokratisches Defizit zu attestieren.

Praktikentheoretische Vorstellungen von Planungsprozessen müssen einem solchen durch administrative Hierarchien geprägten Bild schon deshalb entgegentreten, weil Praktikentheorien die soziale Welt als ein großes Netzwerk von relational und auf einer Ebene miteinander verwobenen Praktiken begreifen (Schatzki 2016a: 32-33; LahrKurten 2018: 324). Als sogenannte flache Ontologien stehen sie unserem Denken in Hierarchiestufen und Maßstabsebenen deshalb sehr kritisch gegenüber (Marston/Jones/ Woodward 2005: 422; Schmid/Reda/Kraehnke et al. 2019: 115-117; Ash 2020: 346-347). Hierarchisch organisierte und als quasi-natürliche Entitäten verinnerlichte Machttopologien, die je nach planerischem Ansatz top-down oder bottom-up durchschritten werden können, sind aus praktikentheoretischer Perspektive demnach nicht denkbar. Aus praktikentheoretischer Sicht gibt es keine hierarchischen Planungsebenen im klassischen Sinn, sondern lediglich unterschiedlich stark miteinander verwobene Bündel von Praktiken und Arrangements, die sich in ihrer Reichweite unterscheiden können (vgl. Schatzki 2016b). Darüber hinaus ist die intentionale Handlungsfähigkeit des Subjekts in Praktikentheorien gegenüber klassischen handlungstheoretischen Zugängen stark eingeschränkt. Demzufolge kann Planung aus einer praktikentheoretischen Perspektive „,nicht als rein rationale Unternehmung verstanden werden, in der durch ,gute Argumente ' auch gute Ergebnisse hervorgebracht werden“ (Geiselhart/Winkler/Dünckmann 2019: 62).

Dünckmann, Haubrich und Runkel (2019) greifen diese 
Einwendungen auf und entwickeln auf deren Basis ein praktikentheoretisch anschlussfähiges Verständnis von Planung. Planungspraktiken heben sich darin als Praktiken zweiter Ordnung aus dem Plenum aller sozialen Praktiken insofern hervor, als dass sie ,in ihrem Vollzug andere, meist alltägliche Praktiken nicht nur miteinbeziehen, sondern diese darüber hinaus als explizites Objekt haben, indem sie auf deren Lenkung, Verhinderung, Ermöglichung, Infragestellung oder Legitimierung abzielen“ (Dünckmann/Haubrich/ Runkel 2019: 317). Planung definiert sich demgemäß weniger über ihre strukturelle Einbettung als vielmehr über die Handlungslogik, die als Bestandteil von Praktiken in der Planungspraxis selbst hinterlegt ist (Dünckmann/Haubrich/ Runkel 2019: 318). Durch geteilte Regeln, Verständnisse und Handlungsketten wird Planung damit selbst $\mathrm{zu}$ einer „site of planning“ (Luukkonen 2017: 272), die sich über ihre topologische Lage im Netz anderer, mit ihr verbundener Praktiken-Arrangement-Bündel definiert. Es stellt sich jedoch die Frage, wo sich im Netz aller Praktiken-Arrangement-Bündel der konkrete, physische Ort von Planungspraktiken befindet. Oder anders ausgedrückt: Findet Planung ausschließlich durch Aushandlungsprozesse zwischen gewählten Politikerinnen und Politikern, Verwaltungsmitarbeiterinnen und Verwaltungsmitarbeitern und ausgewählten Bürgerinnen und Bürgern in den Plenarsälen der Politik, den Konferenzräumen und Büros der Verwaltung oder in organisierten Beteiligungsverfahren statt?

Wie am Beispiel von Treffpunkten der Drogen- und Alkoholkonsumszene in Kiel-Gaarden gezeigt werden konnte, finden gesellschaftliche Grenzziehungsprozesse nicht nur in diesen, typischerweise mit Planungsprozessen assoziierten und mehr oder weniger formalisierten Verwaltungsstrukturen, sondern vor allem im praktischen Handeln auf der Straße statt. Praktiken des gemeinsamen Alkoholkonsums sind gleichzeitig Ausdrucksform von und Katalysator für raumbezogene Identitäten und damit in höchstem Maße politisch. In gewisser Weise schaffen sich die Szenemitglieder den Ort ihres Treffens damit selber. Als Teil von soziomateriellen Arrangements, die in einem konstitutiven Wechselverhältnis zu Planungsprozessen stehen, werden diese Praktiken von der Planung nicht nur verhindert, ermöglicht oder legitimiert, sondern verhindern, ermöglichen oder begrenzen in umgekehrter Richtung selbst die sie betreffenden Planungsprozesse. Ein Verständnis von Planungspraktiken als explizit identifizierbare Praktiken zweiter Ordnung, die auf die Lenkung, Verhinderung, Ermöglichung, Infragestellung oder Legitimierung anderer Praktiken abzielen, greift daher zu kurz und ist am vorliegenden Fall nur bedingt nachzuvollziehen. Vielmehr treten die formellen Planungspraktiken von Politik, Verwaltung und einem Teil der Bürgerschaft mit den informellen und in Teilen widerständigen Planungspraktiken der Drogen- und Alkoholabhängigen in einen inhaltlichen Wettstreit über die soziale und materielle Ausgestaltung von Szenetreffpunkten. Wenn formelle und informelle Planungspraktiken dabei ineinandergreifen - die gewählte Politik beispielsweise einen zuvor von der Verwaltung ausgearbeiteten und durch ein Beteiligungsverfahren legitimierten Bebauungsplan für die vollständige Überplanung eines Szenetreffpunkts beschließen und die Szene anschließend die bislang für diesen Treffpunkt typischen Praktiken einstellen und kampflos das Feld räumen würde -, formen sich Planungskoalitionen, aus denen Raumentwicklung als Ergebnis von in dieselbe Richtung zielenden Praktiken hervorgeht.

\section{Fazit}

Im vorangehenden Kapitel wurde am Beispiel eines Treffpunkts von Drogen- und Alkoholabhängigen in Kiel-Gaarden herausgearbeitet, wie selbst durch so banale Praktiken wie dem kollektiven Alkoholkonsum gesellschaftliche und raumbezogene Grenzziehungen erfolgen, in denen Zugehörigkeit oder Nicht-Zugehörigkeit zum Treffpunkt definiert wird. Die Praktik des gemeinsamen Trinkens trägt daher, wie jede Praktik, ein politisches Moment in sich. Sie unterliegt nicht nur der Steuerung durch formalrechtliche Planungsprozesse, sondern löst selbst ebensolche aus und wird damit zum Teil einer Planungskoalition von Praktiken, aus der Raumentwicklung ko-konstitutiv hervorgeht. Was bedeutet das für Planungen mit Bezug zu Treffpunkten der Drogen- und Alkoholkonsumszene wie auch für Planungsprozesse im Allgemeinen? Aus meiner Sicht muss es gelingen, Planungsprozesse für die praktikentheoretische $\mathrm{Be}$ trachtungsweise zu öffnen. Dabei muss es ganz zentral darum gehen, das in Praktiken eingelagerte Wissen der Praktikerinnen und Praktiker vor Ort zu mobilisieren und in den Prozess einzuspeisen. Dies darf jedoch nicht, wie in derzeitigen Beteiligungsformaten bisweilen zu beobachten, als Feigenblatt dafür dienen, ohnehin schon bestehende Planungsvorhaben zu legitimieren. Vielmehr müssen die vor Ort stattfindenden Praktiken als fundamentaler Teil der Lebenswelt der Ausübenden wie auch als wichtiger Beitrag zum Planungsprozess ernst genommen und in im engeren Sinne fachplanerische Praktiken übersetzt werden. Planerische Instrumente zu entwickeln, die eine solche Übersetzung gewährleisten oder zumindest vereinfachen, wird eine der drängendsten Aufgaben sein, denen sich eine Planungswissenschaft nach dem practice turn in den Sozialwissenschaften stellen muss.

Partizipative Erhebungsmethoden wie das im vorliegenden Fall durchgeführte Fotoprojekt könnten ein solches Instrument sein, wenn sie konsequent auf die Erfassung von Praktiken und deren Weiterverarbeitung im Planungspro- 
zess ausgerichtet werden. In Bezug auf die Treffpunkte der Drogen- und Alkoholabhängigen von Kiel-Gaarden würde die für die Fachplanung verfügbare Datenbasis damit um die Dimension der dort stattfindenden Praktiken erweitert. Das ist insbesondere deshalb relevant, weil diese Praktiken sehr spezifisch sind und sich von den Praktiken an anderen, auf den ersten Blick vielleicht ähnlich erscheinenden Orten unterscheiden können. Ein solches praktikensensibles Verständnis von Planung könnte zugleich eine Verschiebung der Ziele im Umgang mit den Treffpunkten bedeuten. Nimmt man die Bedeutung des gemeinsamen Alkoholkonsums ernst, so dürfte, auch in der Abwägung mit anderen dort stattfindenden Praktiken, nicht mehr (nur) die Verdrängung der Szene, sondern (auch) die Ermöglichung spezifischer Konsumpraktiken das Ziel von Planung sein. Dass soziomaterielle Arrangements im öffentlichen Raum, einem praktikensensiblen Planungsverständnis folgend, ganz bewusst im Hinblick auf die Praktiken ihrer Nutzerinnen und Nutzer entwickelt würden, wäre dann auch das Ergebnis einer Planungskoalition, in der nicht nur für die Menschen, sondern mit den Menschen geplant würde.

Danksagung Der vorliegende Beitrag wurde ganz wesentlich durch meine Mitarbeit im Rahmen des Forschungsnetzwerks „Humangeographische Forschungsperspektiven nach dem practice turn in den Sozialwissenschaften“ der Deutschen Forschungsgemeinschaft (DFG) geprägt. Ich danke den Teilnehmerinnen und Teilnehmern des Netzwerks für die zahlreichen Diskussionen und Anregungen sowie der Deutschen Forschungsgemeinschaft für die Finanzierung der Netzwerkarbeit.

\section{Literatur}

Alkemeyer, T.; Buschmann, N. (2016): Praktiken der Subjektivierung - Subjektivierung als Praxis. In: Schäfer, H. (Hrsg.): Praxistheorie. Ein soziologisches Forschungsprogramm. Bielefeld, 115-136. https://doi.org/ 10.14361/9783839424049-006

Amann, K.; Hirschauer, S. (1997): Die Befremdung der eigenen Kultur. Ein Programm. In: Hirschauer, S.; Amann, K. (Hrsg.): Die Befremdung der eigenen Kultur. Zur ethnographischen Herausforderung soziologischer Empirie. Frankfurt am Main, 7-52.

Ash, J. (2020): Flat Ontology and Geography. In: Dialogues in Human Geography 10, 3, 345-361. https://doi.org/10. 1177/2043820620940052

Bache, I.; Flinders, M. (Hrsg.) (2004): Multi-Level Governance. Oxford. https://doi.org/10.1093/0199259259.001. 0001

Basten, L.; Gerhard, U. (2016): Stadt und Urbanität. In: Freytag, T.; Gebhardt, H.; Gerhard, U.; Wastl-Walter, D. (Hrsg.): Humangeographie kompakt. Berlin, 115-139. https://doi.org/10.1007/978-3-662-44837-3_6
Bischoff, A.; Selle, K.; Sinning, H. (2020): Informieren, Beteiligen, Kooperieren. Kommunikation in Planungsprozessen. Eine Übersicht zu Formen, Verfahren und Methoden. Dortmund.

Bourdieu, P. (1982): Die feinen Unterschiede. Kritik der gesellschaftlichen Urteilskraft. Frankfurt am Main.

Bourdieu, P. (1987): Sozialer Sinn. Kritik der theoretischen Vernunft. Frankfurt am Main.

Collier, J. Jr.; Collier, M. (1986): Visual Anthropology. Photography as a Research Method. Albuquerque.

Dirksmeier, P. (2009): Urbanität als Habitus. Zur Sozialgeographie städtischen Lebens auf dem Land. Bielefeld. https://doi.org/10.14361/9783839411278

Dirksmeier, P. (2013): Zur Methodologie und Performativität qualitativer visueller Methoden - Die Beispiele der Autofotografie und reflexiven Fotografie. In: Rothfuß, E.; Dörfler, T. (Hrsg.): Raumbezogene qualitative Sozialforschung. Wiesbaden, 83-101. https://doi.org/10.1007/ 978-3-531-93240-8_4

Dünckmann, F.; Fladvad, B. (2016): The Practice of Changing the Rules of Practice. An Agonistic View on Food Sovereignty. In: Geographische Zeitschrift 104, 1, 25-49.

Dünckmann, F.; Haubrich, D.; Runkel, S. (2019): Praktiken und Planung. In: Schäfer, S.; Everts, J. (Hrsg.): Handbuch Praktiken und Raum. Humangeographie nach dem Practice Turn. Bielefeld, 317-339. https://doi.org/ 10.14361/9783839446034-013

Everts, J. (2016): Connecting Sites. Practice Theory and Large Phenomena. In: Geographische Zeitschrift 104, 1, 50-67.

Everts, J.; Schäfer, S. (2019): Praktiken und Raum. In: Schäfer, S.; Everts, J. (Hrsg): Handbuch Praktiken und Raum. Humangeographie nach dem Practice Turn. Bielefeld, 7-19. https://doi.org/10.14361/9783839446034-001

Everts, J.; Lahr-Kurten, M.; Watson, M. (2011): Practice Matters! Geographical Inquiry and Theories of Practice. In: Erdkunde 65, 4, 323-334. https://doi.org/10.3112/ erdkunde.2011.04.01

Everts, J.; Geiselhart, K.; Rominger, S.; Winkler, J. (2019): Praktiken des Regierens, dominante Projekte, Verantwortung. Praxeologische Zugänge zum Thema Macht. In: Schäfer, S.; Everts, J. (Hrsg.): Handbuch Praktiken und Raum. Humangeographie nach dem Practice Turn. Bielefeld, 223-243. https://doi.org/10.14361/ 9783839446034-009

Feuerbach, L. (1841): Das Wesen des Christenthums. Leipzig.

Flick, U. (2007): Qualitative Sozialforschung. Eine Einführung. Reinbek.

Fürst, D. (2001): Begriff der Planung, Entwicklung der Planung in Deutschland. In: Fürst, D.; Scholles, F. (Hrsg.): 
Handbuch Theorien und Methoden der Raum- und Umweltplanung. Dortmund, 9-24.

Fürst, D. (2018): Planung. In: ARL - Akademie für Raumforschung und Landesplanung (Hrsg.): Handwörterbuch der Stadt- und Raumentwicklung. Hannover, 1711-1719.

Geertz, C. (1983): Dichte Beschreibung. Beiträge zum Verstehen kultureller Systeme. Frankfurt am Main.

Geiselhart, K.; Winkler, J.; Dünckmann, F. (2019): Vom Wissen über das Tun - praxeologische Ansätze für die Geographie von der Analyse bis zur Kritik. In: Schäfer, S.; Everts, J. (Hrsg.): Handbuch Praktiken und Raum. Humangeographie nach dem Practice Turn. Bielefeld, 21-75. https://doi.org/10.14361/9783839446034-002

Gherardi, S. (2017): Sociomateriality in Posthuman Practice Theory. In: Hui, A.; Schatzki, T. R.; Shove, E. (Hrsg.): The Nexus of Practices. Connections, Constellations, Practitioners. London, 38-51.

Giddens, A. (1997): Die Konstitution der Gesellschaft. Grundzüge einer Theorie der Strukturierung. Frankfurt am Main.

Gläser, J.; Laudel, G. (2006): Experteninterviews und qualitative Inhaltsanalyse als Instrumente rekonstruierender Untersuchungen. Wiesbaden.

Hamedinger, A. (2018): Inkrementalismus / Perspektivischer Inkrementalismus. In: ARL - Akademie für Raumforschung und Landesplanung (Hrsg.): Handwörterbuch der Stadt- und Raumentwicklung. Hannover, 989-994.

Hammersley, M.; Atkinson, P. (2007): Ethnography. Principles in Practice. London. https://doi.org/10.4324/ 9780203944769

Harper, D. (1988): Visual Sociology. Expanding Sociological Vision. In: The American Sociologist 19, 1, 54-70. https://doi.org/10.1007/BF02692374

Healey, P. (1992): Planning through Debate. The Communicative Turn in Planning Theory. In: The Town Planning Review 63, 2, 143-162.

Healey, P. (1996): The Communicative Turn in Planning Theory and its Implications for Spatial Strategy Formation. In: Environment and Planning B: Planning and Design 23, 2, 217-234. https://doi.org/10.1068/b230217

Helfferich, C. (2009): Die Qualität qualitativer Daten. Manual für die Durchführung qualitativer Interviews. Wiesbaden.

Hillebrandt, F. (2009): Praxistheorie. In: Kneer, G.; Schroer, M. (Hrsg.): Handbuch Soziologische Theorien. Wiesbaden, 369-394. https://doi.org/10.1007/978-3-53191600-2_18

Hillebrandt, F. (2016): Die Soziologie der Praxis als post-strukturalistischer Materialismus. In: Schäfer, H. (Hrsg.): Praxistheorie. Ein soziologisches Forschungs- programm. Bielefeld, 71-93. https://doi.org/10.14361/ 9783839424049-004

Hörning, K. H.; Reuter, J. (2004): Doing Culture. Kultur als Praxis. In: Hörning, K. H.; Reuter, J. (Hrsg.): Doing Culture. Neue Positionen zum Verhältnis von Kultur und sozialer Praxis. Bielefeld, 9-15. https://doi.org/10.14361/ 9783839402436-001

Jackson, P.; Brembeck, H.; Everts, J.; Fuentes, M.; Halkier, B.; Hertz, F. D.; Meah, A.; Viehoff, V.; Wenzl, C. (2018): Reframing Convenience Food. London. https://doi.org/ 10.1007/978-3-319-78151-8

Jayne, M.; Holloway, S. L.; Valentine, G. (2006): Drunk and Disorderly. Alcohol, Urban Life and Public Space. In: Progress in Human Geography 30, 4, 451-468. https:// doi.org/10.1191/0309132506ph618oa

Jayne, M.; Valentine, G.; Holloway, S. L. (2010): Emotional, Embodied and Affective Geographies of Alcohol, Drinking and Drunkenness. In: Transactions of the Institute of British Geographers 35, 4, 540-554. https://doi. org/10.1111/j.1475-5661.2010.00401.x

Kraehnke, L. (2014): Das Wohnzimmer unter freiem Himmel? Eine humanistisch-geographische Untersuchung von Treffpunkten der Straßenszene in Kiel-Gaarden. In: Europa Regional 20, 4, 200-213.

Lahr-Kurten, M. (2012): Deutsch sprechen in Frankreich. Praktiken der Förderung der deutschen Sprache im französischen Bildungssystem. Bielefeld. https://doi.org/10. 14361/transcript.9783839420171

Lahr-Kurten, M. (2018): Intensive Geographien - die Versorgung von extremen Frühgeburten in Deutschland aus praxistheoretischer Perspektive. In: Geographica Helvetica 73, 4, 321-333. https://doi.org/10.5194/gh-73-3212018

Langhagen-Rohrbach, C. (2010): Raumordnung und Raumplanung. Darmstadt.

Lapintie, K. (2010): Planning Theory. In: Hutchison, R. (Hrsg.): Encyclopedia of Urban Studies. Thousand Oaks, 603-607.

Lave, J.; Wenger, E. (1991): Situated Learning. Legitimate Peripheral Participation. Cambridge. https://doi.org/10. 1017/CBO9780511815355

LeGates, R. (2005): Urban Planning. In: Caves, R. W. (Hrsg.): Encyclopedia of the City. London, 479-482.

Luukkonen, J. (2017): A Practice Theoretical Perspective on the Europeanization of Spatial Planning. In: European Planning Studies 25, 2, 259-277. https://doi.org/ $10.1080 / 09654313.2016 .1260092$

Marston, S. A.; Jones, J. P.; Woodward, K. (2005): Human Geography Without Scale. In: Transactions of the Institute of British Geographers 30, 4, 416-432. https://doi. org/10.1111/j.1475-5661.2005.00180.x

Marx, K. (1845): Thesen über Feuerbach. In: Marx-Engels- 
Werke 3, 1845-1846. http://www.mlwerke.de/me/me03/ me03_005.htm (09.04.2021).

Mattissek, A.; Prossek, A. (2013): Regieren und Planen. In: Lossau, J.; Freytag, T.; Lippuner, R. (Hrsg.): Schlüsselbegriffe der Kultur- und Sozialgeographie. Stuttgart, 198-211.

Mattissek, A.; Pfaffenbach, C.; Reuber, P. (2013): Methoden der empirischen Humangeographie. Braunschweig.

Meuser, M.; Nagel, U. (2002): ExpertInneninterviews - vielfach erprobt, wenig bedacht. Ein Beitrag zur qualitativen Methodendiskussion. In: Bogner, A.; Littig, B.; Menz, W. (Hrsg.): Das Experteninterview. Theorie, Methode, Anwendung. Wiesbaden, 71-93. https://doi.org/10.1007/ 978-3-322-93270-9

Nicolini, D. (2012): Practice Theory, Work, and Organization. An Introduction. Oxford.

Niesyto, H. (2006): Bildverstehen als mehrdimensionaler Prozess. Vergleichende Auswertung von Bildinterpretationen und methodische Reflexion. In: Marotzki, W.; Niesyto, H. (Hrsg.): Bildinterpretation und Bildverstehen. Methodische Ansätze aus sozialwissenschaftlicher, kunst- und medienpädagogischer Perspektive. Wiesbaden, 253-286. https://doi.org/10.1007/978-3-53190399-6_11

Pink, S. (2012): Situating Everyday Life. Practices and Places. London. https://doi.org/10.4135/9781446250679

Reckwitz, A. (2003): Grundelemente einer Theorie sozialer Praktiken. Eine sozialtheoretische Perspektive. In: Zeitschrift für Soziologie 32, 4, 282-301. https://doi.org/10. 1515/zfsoz-2003-0401

Reckwitz, A. (2016): Kreativität und soziale Praxis. Studien zur Sozial- und Gesellschaftstheorie. Bielefeld. https:// doi.org/10.14361/9783839433454

Reckwitz, A. (2017): Practices and their Affects. In: Hui, A.; Schatzki, T. R.; Shove, E. (Hrsg.): The Nexus of Practices. Connections, Constellations, Practitioners. London, 114-125.

Reda, J.; Runkel, S. (2019): Gewohnheiten und Routinen - praxistheoretische Zugänge zu Geographien des Alltags. In: Schäfer, S.; Everts, J. (Hrsg.): Handbuch Praktiken und Raum. Humangeographie nach dem Practice Turn. Bielefeld, 193-221. https://doi.org/10.14361/ 9783839446034-008

Rhodes, R. A. W. (1996): The New Governance. Governing Without Government. In: Political Studies 44, 4, 652-667. https://doi.org/10.1111/j.1467-9248.1996. tb01747.x

Rose, G. (2016): Visual Methodologies. An Introduction to Researching with Visual Materials. Los Angeles.

Rosol, M.; Dzudzek, I. (2020): Partizipative Planung. In: Belina, B.; Naumann, M.; Strüver, A. (Hrsg.): Handbuch Kritische Stadtgeographie. Münster, 329-334.
Rothfuß, E.; Dörfler, T. (2013): Prolog - Raumbezogene qualitative Sozialforschung. Konzeptionelle Überlegungen zwischen Geographie und Soziologie. In: Rothfuß, E.; Dörfler, T. (Hrsg.): Raumbezogene qualitative Sozialforschung. Wiesbaden, 7-31. https://doi.org/10.1007/ 978-3-531-93240-8_1

Schäfer, S.; Everts, J. (Hrsg.) (2019): Handbuch Praktiken und Raum. Humangeographie nach dem Practice Turn. Bielefeld. https://doi.org/10.14361/9783839446034

Schatzki, T. R. (1996): Social Practices. A Wittgensteinian Approach to Human Activity and the Social. Cambridge.

Schatzki, T. R. (2002): The Site of the Social. A Philosophical Account of the Constitution of Social Life and Change. Pennsylvania.

Schatzki, T. R. (2003): A New Societist Social Ontology. In: Philosophy of the Social Sciences 33, 2, 174-202. https://doi.org/10.1177/0048393103033002002

Schatzki, T. R. (2012): A Primer on Practices. Theory and Research. In: Higgs, J.; Barnett, R.; Billett, S.; Hutchings, M.; Trede, F. (Hrsg.): Practice-Based Education. Perspectives and Strategies. Rotterdam, 13-26. https:// doi.org/10.1007/978-94-6209-128-3_2

Schatzki, T. R. (2016a): Practice Theory as Flat Ontology. In: Spaargaren, G.; Weenink, D.; Lamers, M. (Hrsg.): Practice Theory and Research. Exploring the Dynamics of Social Life. London, 28-42. https://doi.org/10.4324/ 978131565690-12

Schatzki, T. R. (2016b): Keeping Track of Large Phenomena. In: Geographische Zeitschrift 104, 1, 4-24.

Schatzki, T. R. (2017): Sayings, Texts and Discursive Formations. In: Hui, A.; Schatzki, T.R.; Shove, E. (Hrsg.): The Nexus of Practices. Connections, Constellations, Practitioners. London, 126-140.

Schatzki, T. R. (2019): Social Change in a Material World. London. https://doi.org/10.4324/9780429032127

Schatzki, T. R.; Knorr Cetina, K.; von Savigny, E. (Hrsg.) (2001): The Practice Turn in Contemporary Theory. London. https://doi.org/10.4324/9780203977453

Schmid, B.; Reda, J.; Kraehnke, L.; Schwegmann, R. (2019): The Site of the Spatial - eine praktikentheoretische Erschließung geographischer Raumkonzepte. In: Schäfer, S.; Everts, J. (Hrsg.): Handbuch Praktiken und Raum. Humangeographie nach dem Practice Turn. Bielefeld, 93-135. https://doi.org/10.14361/9783839446034004

Selle, K. (2011): ,Particitainment' oder: Beteiligen wir uns zu Tode? Wenn alle das Beste wollen und Bürgerbeteiligung dennoch zum Problem wird. In: pnd online 3, $1-19$.

Shove, E.; Pantzar, M. (2016): Rekrutierung und Reproduktion. Karrieren und Träger von Digitalfotografie und Floorball. In: Schäfer, H. (Hrsg): Praxistheorie. Ein so- 
ziologisches Forschungsprogramm. Bielefeld, 95-113. https://doi.org/10.14361/9783839424049-005

Shove, E.; Pantzar, M.; Watson, M. (2012): The Dynamics of Social Practice. Everyday Life and How it Changes. London. https://doi.org/10.4135/9781446250655

Stoker, G. (1998): Governance as Theory. Five Propositions. In: International Social Science Journal 50, 1, 17-28. https://doi.org/10.1111/issj.12189

Warf, B. (2014): High Points. An Historical Geography of Cannabis. In: Geographical Review 104, 4, 414-438. https://doi.org/10.1111/j.1931-0846.2014.12038.x
Wenger, E. (1998): Communities of Practice. Learning, Meaning, and Identity. Cambridge. https://doi.org/10. 1017/CBO9780511803932

Wiechmann, T. (2018): Planungstheorie. In: ARL - Akademie für Raumforschung und Landesplanung (Hrsg.): Handwörterbuch der Stadt- und Raumentwicklung. Hannover, 1771-1784.

Zimmermann, K. (2017): Praxisansätze in der planungswissenschaftlichen Diskussion. In: Nachrichten der ARL 47, $1,14-16$. 